\title{
Seeking the Important Nodes of Complex Networks in Product R\&D Team Based on Fuzzy AHP and TOPSIS
}

\author{
Wei Zhang, ${ }^{1,2}$ Qingpu Zhang, ${ }^{1,2}$ and Hamidreza Karimi ${ }^{3}$ \\ ${ }^{1}$ School of Management, Harbin Institute of Technology, Harbin 150001, China \\ ${ }^{2}$ HIT-UMKC Institute for Entrepreneurship and Innovation, Harbin Institute of Technology, Harbin 150030, China \\ ${ }^{3}$ Department of Engineering, Faculty of Engineering and Science, University of Agder, 4898 Grimstad, Norway
}

Correspondence should be addressed to Wei Zhang; hbue_wb@163.com

Received 8 August 2013; Accepted 25 August 2013

Academic Editor: Zidong Wang

Copyright (C) 2013 Wei Zhang et al. This is an open access article distributed under the Creative Commons Attribution License, which permits unrestricted use, distribution, and reproduction in any medium, provided the original work is properly cited.

\begin{abstract}
How to seek the important nodes of complex networks in product research and development (R\&D) team is particularly important for companies engaged in creativity and innovation. The previous literature mainly uses several single indicators to assess the node importance; this paper proposes a multiple attribute decision making model to tentatively solve these problems. Firstly, choose eight indicators as the evaluation criteria, four from centralization of complex networks: degree centrality, betweenness centrality, closeness centrality, and eigenvector centrality and four from structural holes of complex networks: effective size, efficiency, constraint, and hierarchy. Then, use fuzzy analytic hierarchy process (AHP) to obtain the weights of these indicators and use technique for order preference by similarity to an ideal solution (TOPSIS) to assess the importance degree of each node of complex networks. Finally, taking a product R\&D team of a game software company as a research example, test the effectiveness, operability, and efficiency of the method we established.
\end{abstract}

\section{Introduction}

All innovations begin as creative ideas or solutions, especially for product innovation; it is critical to generate creative ideas and develop them to the novel, valuable, and practical creative solutions in the frond-end R\&D process [1]. Due to the limited knowledge and information of a single individual, it is often difficult to form the creative solution which can meet the new product needs. In actual product $\mathrm{R} \& \mathrm{D}$ process, the product $\mathrm{R} \& \mathrm{D}$ team is the effective and basic work unit for finishing these works. Almost all companies such as Shell, Nortel, and Gamble have established the product R\&D teams to generate new paradigm-breaking ideas and solutions in the frond-end innovation process [2]. As the important nodes in the complex network of product R\&D team, some members such as the organization power leaders, the opinion leaders, or the key figures in the interpersonal relationship network can affect the whole creative process and the final results. However, However, it is a difficulty in the creative solution formation process of product R\&D team that to seek and find out the important members and reasonably treat and manage them.

The nonhomogenous nature of complex network's topological structure decides the large differences in the important degree of each node in the network [3]; furthermore, the scale-freeness of complex networks always leads to the dual characteristics of robustness and fragility [4]. Lai et al. (2004) regard that the whole network would be paralyzed if $5 \%-10 \%$ important nodes fail [5]. Hence, to accurately distinguish, seek, and pick out the important nodes of complex networks has significant theoretical and practical significances and receives wide attention from many scholars. Chen et al. (2004) analyzed the important nodes of criminal relationship network which can distinguish core members and the general members in criminal gangs [6]; Golbeck and Hendler (2004) used a ranking method based on reputation values to assess the nodes importance of email communication network [7], and Shetty and Adibi (2005) established an entropy model which combined cross-entropy and text mining technology 
to seek the important nodes in the same type of complex network [8]. In addition, other scholars assessed the nodes importance of social networks [9], R\&D cooperation networks [10], transportation network [11], worldwide web network [12], rumor proliferation networks [13], and so on.

Currently, there are so many methods used to assess the important nodes of complex networks [14-20], which are derived from graph theory [21] and graph-based data mining [22] and can be grouped into three perspectives: social network analysis method, system science analysis method, and information search field analysis method. Social network analysis is based on a hypothesis that the importance of the node is equivalent to the significance generated by the connection between this node with other nodes [23]. The basic idea of this analysis method is that the difference of the nodes importance in the network can be obtained by analyzing some useful information, such as the degree of the node, the shortest path, and the edge node weights [24]. System science analysis method is to reflect the integrity of the system's certain function by using network connectivity and to reflect the network node (set) importance by measuring the extent of damage in the network connectivity when the node is deleted [24]. The basic idea is based on the equivalence of destruction and importance [25]. Information search field analysis method views the Internet as a huge figure, in which nodes represent pages and (directed) edges represent hyperlinks between pages. PageRank [26] and HITS [27] are two of the most famous and the most representative algorithms in this type of methods. With the special network background, the activities of social interactions and exchanges are particularly prominent in creative solution formation process of product R\&D team. The central figures can directly affect other members' behaviors and thinks to generate creative ideas or develop them into creative solutions. Centralization and structural holes of complex networks can quantify the importance of individual in team [28]. So we will seek and pick out the important nodes (key members) of complex network (product R\&D team) by centralization indicators and structural holes indicators. Common centralization indicators are degree centrality, betweenness centrality, closeness centrality, and eigenvector centrality. Common structural holes indicators are effective size, efficiency, constraint, and hierarchy, in which constraint is the most important indicators. In this paper, we choose these eight indicators as the decision criteria to determine the nodes importance by fuzzy AHP and TOPSIS.

\section{Definitions of Decision Criteria}

Complex network can be represented by using figure $G=$ $(V, E)$, where $G$ is an undirected connected graph with $n$ nodes and $m$ edges, $V=\left\{v_{1}, v_{2}, v_{3}, \ldots, v_{n}\right\}$ represents the set of nodes, and $E=\left\{e_{1}, e_{2}, e_{3}, \ldots, e_{m}\right\} \subseteq V \times V$ represents the set of edges. $A=\left[a_{i j}\right]$ is $G$ 's adjacency matrix with $n$ rows and $n$ columns; element $a_{i j}$ in $A$ can be defined as follows:

$$
a_{i j}= \begin{cases}1, & \text { Node } i \text { and node } j \text { are adjacent } \\ 0, & \text { Node } i \text { and node } j \text { are not adjacent. }\end{cases}
$$

Here is the definitions of indicators we select.
2.1. Degree Centrality. Degree centrality is the basic parameters used to study the scale-free network topology, which can describe the direct impact by nodes in the static network; its value is the number of nodes directly connected by this node.

The degree centrality $C_{d}(v)$ of node $v$ is

$$
C_{d}(v)=d(v),
$$

where $d(v)$ is the degree of node $v$, which is defined as the number of ties that a node has.

In order to compare the centrality of different nodes, normalize the degree indicator:

$$
C_{D}(v)=\frac{C_{d}(v)}{n-1}
$$

Degree centrality reflects the ability of the node to directly obtain network flow content. The larger its value, the more important the node in the network.

2.2. Betweenness Centrality. Betweenness centrality characterizes influence of the network nodes on information flow. The betweenness centrality of node $v$ is

$$
C_{B}(v)=\sum_{j<k} \frac{g_{j k}(v)}{g_{j k}},
$$

where $g_{j k}$ is the number of the shortest paths from $j$ to $k$ and $g_{j k}(v)$ is the number of the shortest paths from $j$ to $k$ that pass through a node $v$. The largest betweenness of node $v$ is $(n-1)(n-2) / 2$; normalize the betweenness indicator:

$$
C_{B}(v)=\frac{C_{b}(v)}{[(n-1)(n-2) / 2]} .
$$

Betweenness centrality can describe the important ability of nodes controlling the information flow between other nodes. The larger the betweenness centrality value of a node, the more information and other resources this node has and the more important the node in the network.

2.3. Closeness Centrality. Closeness centrality characterizes the degree of difficulty that a node over the network has in connecting to other network nodes, and its value is defined as reciprocal of the sum of distances between the node and all other nodes.

The closeness centrality of node $v$ is

$$
C_{c}(v)=\left[\sum_{y=1}^{n} d_{v y}\right]^{-1}
$$

where $d_{v y}$ is the shortest path between node $v$ and node $y$.

As the sum of the distances between the node and all other nodes cannot be less than $n-1$, normalize the closeness indicator:

$$
C_{c}(v)=(n-1) C_{c}(v) .
$$

Closeness centrality can be regarded as a measure of how long the information can spread from a given node to other reachable nodes in the network. The smaller the value, the smaller the time spread. 
TABLE 1: Definition and membership function of fuzzy scale.

\begin{tabular}{lccc}
\hline $\begin{array}{l}\text { Intensity of } \\
\text { importance }\end{array}$ & $\begin{array}{c}\text { Fuzzy } \\
\text { number }\end{array}$ & Definition & $\begin{array}{c}\text { Membership } \\
\text { function }\end{array}$ \\
\hline 9 & $\widetilde{9}$ & Extreme importance & $(8,9,10)$ \\
7 & $\widetilde{7}$ & Very strong importance & $(6,7,8)$ \\
5 & $\widetilde{5}$ & Strong importance & $(4,5,6)$ \\
3 & $\widetilde{3}$ & Moderate importance & $(2,3,4)$ \\
1 & $\widetilde{1}$ & Equal importance & $(1,1,2)$ \\
\hline
\end{tabular}

2.4. Eigenvector Centrality. Eigenvector centrality takes into account the linear relationship between one node's centrality indicators and the centrality indicators of other nodes around, which is the linear superposition of the centrality values of its adjacent nodes.

The eigenvector centrality of node $v$ is

$$
e_{v}=\lambda^{-1} \sum_{j=1}^{n} A_{v j} e_{j}
$$

where $e_{v}$ denotes the score of the node $v, A$ is the adjacency matrix of the network, $n$ is the total number of nodes, and $\lambda$ is a constant. In vector notation, this can be rewritten as $E=\lambda^{-1} A X$ or as the eigenvector equation $A X=\lambda E$.

Structural holes can be defined as nonredundant links between two nodes in the network [29]; that is, if one node links together two separate nodes, the network location of this node is the structure holes. It can be measured by the following indicators.

2.5. Effective Size and Efficiency. The effective size of one node is equal to the size of ego network minus the redundancy of network.

The effective size of node $v$ is

$$
\sum_{j}\left(1-\sum_{q} p_{v q} m_{j q}\right), \quad q \neq i, j,
$$

where $j$ is the node connected to $v, q$ is the third node in addition to $v$ and $j$, and $p_{v q} m_{j q}$ is the redundancy between $v$ and $j$, in which $p_{v q}$ is the proportion that $v$ inputs on $q$, and $m_{j q}$ is the marginal strength of the relationship between $j$ and $q$.

The efficiency of a node is equal to the value through dividing effective size by actual size.

2.6. Constraint. Constraint can be defined as the ability of the node to use structural holes in the network. The constrain of node $v$ is

$$
C_{v}=\sum_{j}\left(p_{v j} \sum_{q \neq v \neq j} P_{v q} P_{q j}\right)^{2}
$$

where $q$ is the indirect node which links the $v$ and $j, p_{v j}$ is the ratio of the time or energy taken by $v$ to the total of time or energy, which is the direct input, while $\sum_{q \neq v \neq j} P_{v q} P_{q j}$ is the indirect input. The smaller the value of constraint is, the more important the position of the node is.

2.7. Hierarchy. Hierarchy describes the degree of concentration of constraint on one node, which can be calculated by Coleman-Theil disorder index:

$$
H=\frac{\sum_{j}\left(C_{v j} /(C / N)\right) \ln \left(C_{v j} /(C / N)\right)}{N \ln (N)},
$$

where $N$ is the ego-network size of node $v ; C / N$ is the mean of every node's constraint and the denominator $N \ln (N)$ is the maximum possible sum. If the constraints of one node's all contacts are the same, the hierarchy of this node is zero. Conversely, if all constraints are concentrated on one node, value of this indicator is 1 , which is the maximum.

\section{Comprehensive Evaluation Method Based on Fuzzy AHP and TOPSIS}

3.1. Fuzzy AHP Methodology. According to the research background and definitions of each indicator, we apply the fuzzy AHP to get different indicator's weights. In this paper, we use triangular fuzzy numbers [30]; a fuzzy number is a special fuzzy set $A=\{(x, \mu \widetilde{A}(x)), x \in R\}$ and $\mu \widetilde{A}(x)$ is a continuous mapping from $R$ to the closed interval $[0,1]$. A triangular fuzzy number denoted as $\widetilde{A}=(l, m, u)$, where $l \leq m \leq u, l$ and $u$ are, respectively, the lower and upper bounds of $\widetilde{A}$, and $m$ is the modal value of $\widetilde{A}$, has the following triangular-type membership function:

$$
\mu_{\widetilde{A}}(x)= \begin{cases}\frac{(x-l)}{(m-l)}, & l \leq x \leq m, \\ \frac{(u-x)}{(u-m)}, & m \leq x \leq u, \\ 0, & x<l \text { or } x>u .\end{cases}
$$

We will use linguistic variables shown in Table 1 to compare the relative importance of each evaluation indicator by five basic linguistic terms [31], as "equal importance," "moderate importance," "strong importance," "very strong importance," and "extreme importance." $[32,33]$

The procedure of fuzzy AHP is composed of three steps

Step 1. Construct pairwise comparison matrices among all indicators: degree centrality, betweenness centrality, closeness centrality, eigenvector centrality, effective size, efficiency, constraint, and hierarchy. And assign linguistic terms to the 
pairwise comparisons by asking which is more important of each two indicators, such as

$$
\begin{aligned}
\widetilde{A} & =\left[\begin{array}{cccc}
1 & \tilde{a}_{12} & \cdots & \tilde{a}_{1 n} \\
\tilde{a}_{21} & 1 & \cdots & \tilde{a}_{2 n} \\
\vdots & \vdots & \ddots & \vdots \\
\tilde{a}_{n 1} & \tilde{a}_{n 2} & \cdots & 1
\end{array}\right] \\
& =\left[\begin{array}{cccc}
1 & \tilde{a}_{12} & \cdots & \tilde{a}_{1 n} \\
\frac{1}{\tilde{a}_{12}} & 1 & \cdots & \tilde{a}_{2 n} \\
\vdots & \vdots & \ddots & \vdots \\
\frac{1}{\tilde{a}_{1 n}} & \frac{1}{\tilde{a}_{2 n}} & \cdots & 1
\end{array}\right],
\end{aligned}
$$

where

$$
\widetilde{a}_{i j}= \begin{cases}\widetilde{1}, \widetilde{3}, \widetilde{5}, \widetilde{7}, \widetilde{9} & i>j \\ 1 & i=j \\ \widetilde{1}^{-1}, \widetilde{3}^{-1}, \widetilde{5}^{-1}, \widetilde{7}^{-1}, \widetilde{9}^{-1} & i<j\end{cases}
$$

Step 2. Define the fuzzy geometric mean and fuzzy weights of each indicator using geometric mean technique by Buckley (1985) [34] as follows:

$$
\begin{gathered}
\tilde{r}_{i}=\left(\widetilde{a}_{i 1} \otimes \tilde{a}_{i 2} \otimes \cdots \otimes \widetilde{a}_{i n}\right)^{1 / n}, \\
\widetilde{w}_{i}=\widetilde{r}_{i} \otimes\left(\widetilde{r}_{1} \oplus \cdots \oplus \widetilde{r}_{n}\right)^{-1},
\end{gathered}
$$

where $\tilde{a}_{i n}$ is fuzzy comparison value from criterion $i$ to criterion $n$; thus, $\widetilde{r}_{i}$ is geometric mean of fuzzy comparison value from criterion $i$ to each criterion and $\widetilde{w}_{i}$ is the fuzzy weight of the $i$ th criterion, $\widetilde{w}_{i}=\left(l w_{i}, m w_{i}, u w_{i}\right)$.

Here $l w_{i}, m w_{i}$, and $u w_{i}$, respectively, stand for the lower, middle, and the upper values of the fuzzy weight of the $i$ th criterion.

Step 3 (Defuzzification). The procedure of defuzzification is to locate the best nonfuzzy performance value (BNP). We can use the following equation to calculate the BNP value of the fuzzy number:

$$
\mathrm{BNP}_{i}=\frac{\left[\left(u w_{i}-l w_{i}\right)+\left(m w_{i}-l w_{i}\right)\right]}{3}+l w_{i} .
$$

3.2. TOPSIS Methodology. Technique for order preference by similarity to an ideal solution (TOPSIS) is based on the concept that the chosen alternative should have the shortest distance from the positive-ideal solution and the longest distance from the negative-ideal solution. We will use this method to rank the node importance of complex networks, which is composed of seven steps as follows.

Step 1 (Construct the Decision Matrix). Suppose there are $m$ nodes in complex network; the nodes set is $A=$ $\left\{A_{1}, A_{2}, \ldots, A_{m}\right\}$; there are $n$ evaluation indicators to assess the importance of nodes; the indicators set is $C=$ $\left\{C_{1}, C_{2}, \ldots, C_{n}\right\} . A_{i}\left(C_{j}\right)$ is the $i$ th node performance on $j$ th indicator $(i=1,2, \ldots, m ; j=1,2, \ldots, n)$; the decision matrix is

$$
X=\left(\begin{array}{ccc}
A_{1}\left(C_{1}\right) & \cdots & A_{1}\left(C_{n}\right) \\
\vdots & \ddots & \vdots \\
A_{m}\left(S_{1}\right) & \cdots & A_{m}\left(S_{n}\right)
\end{array}\right) .
$$

Step 2 (Normalize the Decision Matrix). There are some complex relationships between indicators, and the dimension of each indicators may be different, so we need to normalize the indicators. of eight indicators of this study, six indicators such as degree centrality, betweenness centrality, closeness centrality, eigenvector centrality, effective size, and efficiency belong to the benefit type indicators, while two indicators, constraint and hierarchy, belong to the cost type indicators. We use the following method to normalize the decision matrix

$$
r_{i j}=\frac{A_{i}\left(C_{j}\right)}{A_{i}\left(C_{j}\right)^{\mathrm{max}}}
$$

if the indicator belongs to benefit type and use the following method to normalize the decision matrix

$$
r_{i j}=\frac{A_{i}\left(C_{j}\right)^{\mathrm{min}}}{A_{i}\left(C_{j}\right)}
$$

if the indicator belongs to cost type, where $A_{i}\left(C_{j}\right)^{\max }=$ $\max \left\{A_{i}\left(C_{j}\right) \mid 1 \leq i \leq m\right\}, A_{i}\left(C_{j}\right)^{\min }=\min \left\{A_{i}\left(C_{j}\right) \mid 1 \leq\right.$ $i \leq m\}$, and the normalized decision matrix is

$$
R=\left(r_{i j}\right)_{n \times m}, \quad i=1,2, \ldots, m ; j=1,2, \ldots, n .
$$

Step 3. On the basis of normalized decision matrix, construct the weighted normalized decision matrix as the following formula:

$$
V=\left[v_{i j}\right]_{n \times n}, \quad i=1,2, \ldots, m ; j=1,2, \ldots, n,
$$

where $v_{i j}=r_{i j} \otimes \omega_{i j}$.

Step 4. Determine the positive-ideal node and negative-ideal node:

$$
\begin{aligned}
& A^{+}=\left\{\max _{i \in L}\left(v_{i 1}, v_{i 2}, \ldots, v_{i n}\right)\right\}=\left\{v_{1}^{\max }, v_{2}^{\max }, \ldots, v_{n}^{\max }\right\}, \\
& A^{-}=\left\{\min _{i \in L}\left(v_{i 1}, v_{i 2}, \ldots, v_{i n}\right)\right\}=\left\{v_{1}^{\min }, v_{2}^{\min }, \ldots, v_{n}^{\min }\right\},
\end{aligned}
$$

where $L=(1,2, \ldots, m)$.

Step 5. Calculate the distance of each node from the positiveideal node and negative-ideal node:

$$
\begin{aligned}
& d_{i}^{+}=\sum_{j=1}^{n} d\left(v_{i j}, v_{j}^{+}\right), \quad i=1,2, \ldots, m ; j=1,2, \ldots, n, \\
& d_{i}^{-}=\sum_{j=1}^{n} d\left(v_{i j}, v_{j}^{-}\right), \quad i=1,2, \ldots, m ; j=1,2, \ldots, n .
\end{aligned}
$$




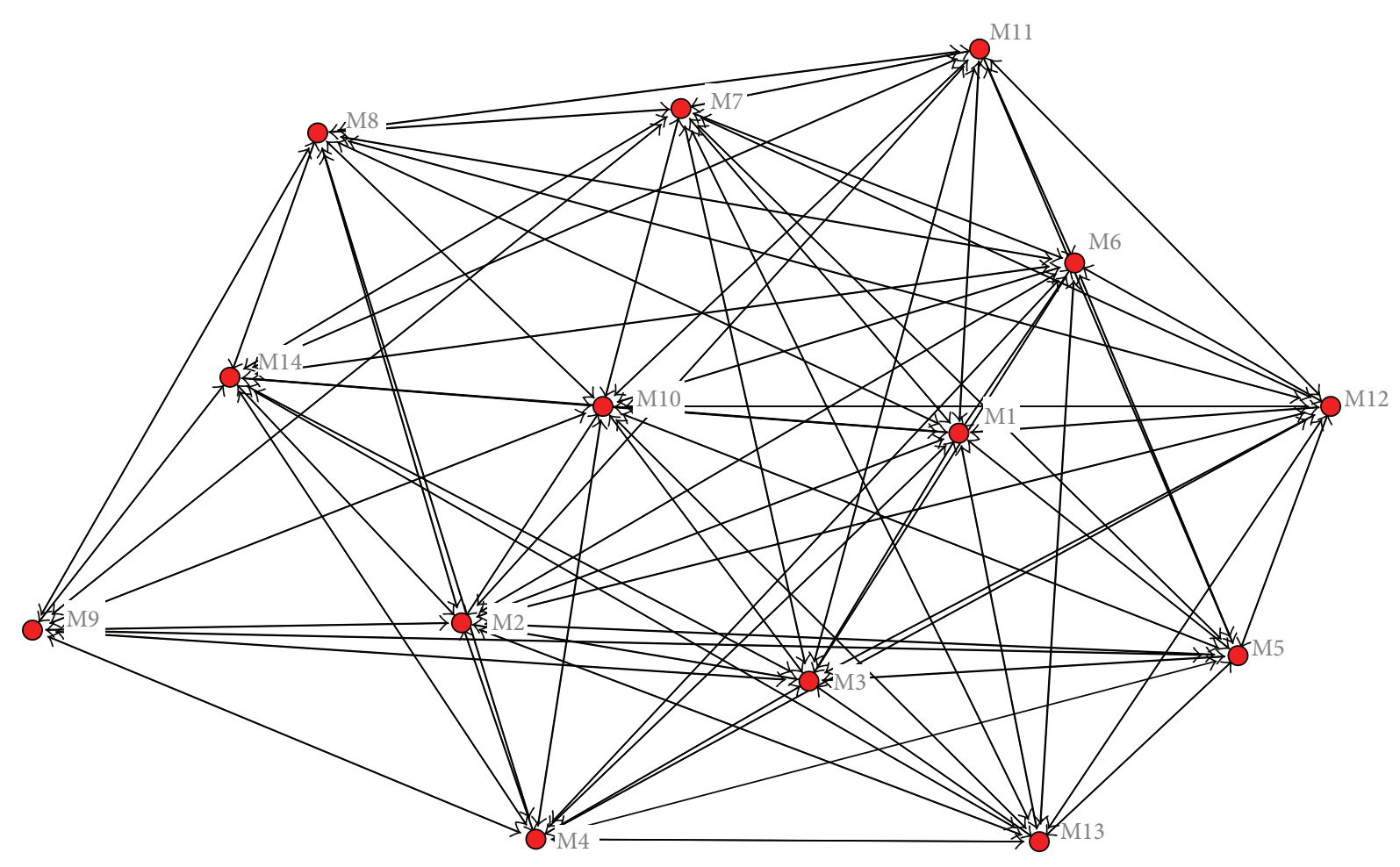

FIgURE 1: Network structure of 14 members.

Step 6. Obtain the closeness coefficients:

$$
Z_{i}=\frac{d_{i}^{-}}{d_{i}^{+}+d_{i}^{-}}=1-\frac{d_{i}^{+}}{d_{i}^{+}+d_{i}^{-}}, \quad i=1,2, \ldots, m,
$$

where we define $d_{i}^{-} /\left(d_{i}^{+}+d_{i}^{-}\right)$as satisfaction degree of $i$ th node and $d_{i}^{+} /\left(d_{i}^{+}+d_{i}^{-}\right)$as gap degree of $i$ th node. According to the descending order of $Z_{i}$, we can determine the ranking order of all nodes and and select the best ones from a set of feasible nodes [35]. The larger the closeness coefficient of one node is, the more important the node is.

\section{Example and Analysis}

In our study, we choose a product $\mathrm{R} \& \mathrm{D}$ team coming from one game software company as a research example. There are 14 members in this team, and the members always communicate with each other in the creative activities. In order to get the accurate data from the members and team, we collect data from different paths and methods, mainly including three paths: questionnaire, email and other social media records, and daily face-to-face meeting records. The data collected by the latter two methods is objective; we can get it by directly collating existing records. The data collected by the questionnaire is subjective, and the data provided by one pair of investigators may be different. For example, when we asked the investigator $A(B)$ to recall the number that he/she communicated with member $B(A)$ outside of work hours, the answers from $A$ and $B$ may be different. So we need to interview repeatedly each pair of investigators and get the final consistent data. The period of data we collect is one month. We finally got the statistical results about the exchange numbers of 14 members as shown in Table 2 and the network structure as shown in Figure 1.

Firstly, we should get the weights of eight indicators by using the fuzzy AHP method. We construct pairwise comparison matrix as follows:

$$
\widetilde{A}=\left[\begin{array}{cccccccc}
1 & \widetilde{3}^{-1} \widetilde{3}^{-1} & 1 & \widetilde{3}^{-1} & 1 & \widetilde{3}^{-1} & 1 & \widetilde{3} \\
\widetilde{3} & 1 & 1 & \widetilde{3} & 1 & 1 & \widetilde{3}^{-1} & 1 \\
1 & 1 & 1 & \widetilde{3} & \widetilde{3}^{-1} & \widetilde{3}^{-1} & 1 & \widetilde{3} \\
\widetilde{3} & \widetilde{3}^{-1} & \widetilde{3}^{-1} & 1 & \widetilde{3}^{-1} & \widetilde{3}^{-1} & \widetilde{3} & 1 \\
1 & 1 & \widetilde{3} & \widetilde{3} & 1 & 1 & \widetilde{3} & 1 \\
\widetilde{3} & 1 & \widetilde{3} & \widetilde{3} & 1 & 1 & \widetilde{5} & 1 \\
1 & \widetilde{3} & 1 & \widetilde{3}^{-1} & \widetilde{3}^{-1} & \widetilde{5}^{-1} & 1 & \widetilde{3}^{-1} \\
\widetilde{3}^{-1} & 1 & \widetilde{3}^{-1} & 1 & 1 & 1 & \widetilde{3} & 1
\end{array}\right]
$$

Use (15) to obtain the fuzzy geometric mean of eight indicators and use (16) to obtain the fuzzy weights of each indicator. Take $\widetilde{r}_{1}$ as the example:

$$
\begin{aligned}
\widetilde{r}_{1}= & \left(\widetilde{a}_{11} \otimes \widetilde{a}_{12} \otimes \widetilde{a}_{13} \otimes \widetilde{a}_{14} \otimes \widetilde{a}_{15} \otimes \widetilde{a}_{16} \otimes \widetilde{a}_{17} \otimes \widetilde{a}_{18}\right)^{1 / 8} \\
= & \left(\left(1 \times 2 \times 1 \times 2 \times 1 \times 2 \times 1 \times \frac{1}{4}\right)^{1 / 8},\right. \\
& \left(1 \times 3 \times 1 \times 3 \times 1 \times 3 \times 1 \times \frac{1}{3}\right)^{1 / 8},
\end{aligned}
$$


TABLE 2: Statistical data of members exchange.

\begin{tabular}{lccccccccccccccc}
\hline & M1 & M2 & M3 & M4 & M5 & M6 & M7 & M8 & M9 & M10 & M11 & M12 & M13 & M14 \\
\hline M1 & 0 & 2 & 7 & 9 & 11 & 12 & 3 & 4 & 0 & 9 & 3 & 9 & 8 & 2 \\
M2 & 2 & 0 & 9 & 10 & 8 & 13 & 0 & 2 & 2 & 9 & 3 & 8 & 12 & 10 \\
M3 & 7 & 9 & 0 & 7 & 6 & 9 & 2 & 0 & 4 & 5 & 3 & 9 & 9 & 9 \\
M4 & 9 & 10 & 7 & 0 & 6 & 15 & 0 & 3 & 2 & 11 & 0 & 5 & 7 & 8 \\
M5 & 11 & 8 & 6 & 6 & 0 & 3 & 7 & 0 & 2 & 2 & 3 & 3 & 8 & 0 \\
M6 & 12 & 13 & 9 & 15 & 3 & 0 & 5 & 11 & 0 & 12 & 2 & 11 & 9 & 10 \\
M7 & 3 & 0 & 2 & 0 & 7 & 5 & 0 & 5 & 9 & 10 & 8 & 2 & 4 \\
M8 & 4 & 2 & 0 & 3 & 0 & 11 & 5 & 0 & 9 & 14 & 12 & 8 & 0 \\
M9 & 0 & 2 & 4 & 2 & 2 & 0 & 9 & 9 & 0 & 5 & 0 & 0 & 0 \\
M10 & 9 & 9 & 5 & 11 & 2 & 12 & 10 & 14 & 5 & 0 & 9 & 7 & 11 & 11 \\
M11 & 3 & 3 & 3 & 0 & 3 & 2 & 8 & 12 & 0 & 9 & 0 & 2 & 0 \\
M12 & 9 & 8 & 9 & 5 & 3 & 11 & 2 & 8 & 0 & 7 & 2 & 0 & 9 \\
M13 & 8 & 12 & 9 & 7 & 8 & 9 & 4 & 0 & 0 & 11 & 0 & 9 & 0 \\
M14 & 2 & 10 & 9 & 8 & 0 & 10 & 2 & 6 & 2 & 11 & 7 & 0 & 2 \\
\hline
\end{tabular}

$$
\begin{aligned}
& \left.\left(2 \times 4 \times 2 \times 4 \times 2 \times 4 \times 2 \frac{1}{2}\right)^{1 / 8}\right) \\
= & (1.091,1.316,2.181) .
\end{aligned}
$$

Similarly, we can obtain the other indicators' fuzzy geometric means as follows:

$$
\begin{aligned}
& \tilde{r}_{2}=(0.771,0.872,1.542) ; \quad \tilde{r}_{3}=(0.841,1,1.682) ; \\
& \widetilde{r}_{4}=(1,1.316,2) ; \quad \widetilde{r}_{5}=(0.565,0.621,1) ; \\
& \widetilde{r}_{6}=(0.475,0.542,0.917) ; \quad \widetilde{r}_{7}=(1.297,1.609,2.502) ; \\
& \tilde{r}_{8}=(1,1.147,2) .
\end{aligned}
$$

The fuzzy weights of $\widetilde{w}_{1}$ as the example are as follows:

$$
\begin{aligned}
\widetilde{w}_{1}= & \tilde{r}_{1} \otimes\left(\widetilde{r}_{1} \oplus \tilde{r}_{2} \oplus \tilde{r}_{3} \oplus \tilde{a}_{14} \oplus \tilde{a}_{15} \oplus \tilde{a}_{16} \oplus \tilde{a}_{17} \oplus \tilde{a}_{18}\right)^{-1} \\
= & (1.091,1.316,2.181) \\
& \otimes\left(\frac{1}{(2.181+1.542+\cdots+2)},\right. \\
& \left.\frac{1}{(1.316+0.872+\cdots+0.1 .147)}\right) \\
= & (0.072,0.156,0.310) .
\end{aligned}
$$

Similarly, we can obtain the other indicators' fuzzy weights as follows:

$$
\begin{array}{lll}
\widetilde{w}_{2}=(0.056,0.104,0.219) ; & \widetilde{w}_{3}=(0.061,0.119,0.239) ; \\
\widetilde{w}_{4}=(0.072,0.156,0.284) ; & \widetilde{w}_{5}=(0.041,0.074,0.142) ; \\
\widetilde{w}_{6}=(0.034,0.064,0.130) ; & \widetilde{w}_{7}=(0.094,0.191,0.355) ; \\
\widetilde{w}_{8}=(0.072,0.136,0.284) . &
\end{array}
$$

Use COA method to take the BNP value of the weight of "degree centrality" indicator as the example; the calculation process is as follows:

$$
\begin{aligned}
\mathrm{BNP}_{w 1} & =\frac{\left[\left(U_{w 1}-L_{w 1}\right)+\left(M_{w 1}-L_{w 1}\right)\right]}{3}+L_{w 1} \\
& =\frac{[(0.310-0.156)+(0.072-0.156)]}{3}+0.156 \\
& =0.179 .
\end{aligned}
$$

Similarly, we can obtain the other dimensions' BNP values in the first stage's evaluation criteria system as follows:

$$
\begin{array}{lll}
\mathrm{BNP}_{w 2}=0.126 ; & \mathrm{BNP}_{w 3}=0.140 ; & \mathrm{BNP}_{w 4}=0.171 ; \\
\mathrm{BNP}_{w 5}=0.086 ; & \mathrm{BNP}_{w 6}=0.076 ; & \mathrm{BNP}_{w 7}=0.213 ; \\
\mathrm{BNP}_{w 8}=0.164 . & &
\end{array}
$$

As the sum of eight indicators' BNP is 1.155, we normalize them and get their weights:

$$
\begin{array}{lll}
w_{1}=0.155 ; & w_{2}=0.019 ; & w_{3}=0.121 ; \\
w_{4}=0.148 ; & w_{5}=0.074 ; & w_{6}=0.067 ; \\
w_{7}=0.184 ; & w_{8}=0.142 . &
\end{array}
$$


TABLE 3: The performance values of eight indicators of 14 team members.

\begin{tabular}{lcccccccc}
\hline & DC & BC & CC & EC & ES & EF & CO & HI \\
\hline M1 & 40.513 & 1.217 & 92.857 & 39.164 & 6.079 & 0.507 & 0.332 & 0.077 \\
M2 & 45.128 & 1.842 & 92.857 & 43.790 & 6.332 & 0.528 & 0.338 \\
M3 & 40.513 & 1.656 & 92.857 & 38.005 & 6.511 & 0.543 & 0.330 \\
M4 & 42.564 & 1.374 & 86.667 & 43.059 & 5.193 & 0.472 & 0.366 \\
M5 & 30.256 & 1.400 & 86.667 & 27.655 & 5.784 & 0.526 & 0.357 & 0.068 \\
M6 & 57.436 & 1.217 & 92.857 & 54.309 & 6.401 & 0.533 & 0.314 \\
M7 & 29.231 & 1.700 & 86.667 & 24.472 & 6.280 & 0.571 & 0.348 \\
M8 & 37.949 & 1.202 & 81.250 & 33.999 & 5.645 & 0.565 & 0.063 \\
M9 & 17.949 & 0.644 & 72.222 & 15.260 & 4.608 & 0.576 & 0.483 \\
M10 & 58.974 & 2.087 & 100.000 & 52.056 & 7.481 & 0.575 & 0.076 \\
M11 & 26.667 & 0.644 & 81.250 & 24.011 & 5.340 & 0.534 & 0.093 \\
M12 & 37.436 & 0.961 & 86.667 & 37.437 & 5.498 & 0.500 & 0.127 \\
M13 & 40.513 & 0.501 & 81.250 & 40.314 & 4.549 & 0.455 & 0.363 \\
M14 & 35.385 & 1.505 & 86.667 & 35.143 & 5.648 & 0.513 & 0.378 & 0.114 \\
\hline
\end{tabular}

DC: degree centrality, BC: betweenness centrality, CC: closeness centrality, EC: eigenvector centrality, ES: effective size, EF: efficiency, CO: constraint, and HI: hierarchy.

TABLE 4: The normalized results of eight indicators of 14 team members.

\begin{tabular}{|c|c|c|c|c|c|c|c|c|}
\hline & DC & $\mathrm{BC}$ & CC & EC & ES & $\mathrm{EF}$ & $\mathrm{CO}$ & HI \\
\hline M1 & 0.687 & 0.583 & 0.929 & 0.721 & 0.813 & 0.880 & 0.855 & 0.597 \\
\hline M2 & 0.765 & 0.883 & 0.929 & 0.806 & 0.846 & 0.917 & 0.840 & 0.495 \\
\hline M3 & 0.687 & 0.793 & 0.929 & 0.700 & 0.870 & 0.943 & 0.861 & 0.676 \\
\hline M4 & 0.722 & 0.658 & 0.867 & 0.793 & 0.694 & 0.819 & 0.776 & 0.505 \\
\hline M5 & 0.513 & 0.671 & 0.867 & 0.509 & 0.773 & 0.913 & 0.796 & 0.730 \\
\hline M6 & 0.974 & 0.583 & 0.929 & 1.000 & 0.856 & 0.925 & 0.904 & 0.754 \\
\hline M7 & 0.496 & 0.815 & 0.867 & 0.451 & 0.839 & 0.991 & 0.816 & 0.605 \\
\hline M8 & 0.643 & 0.576 & 0.813 & 0.626 & 0.755 & 0.981 & 0.759 & 0.495 \\
\hline M9 & 0.304 & 0.309 & 0.722 & 0.281 & 0.616 & 1.000 & 0.582 & 0.362 \\
\hline M10 & 1.000 & 1.000 & 1.000 & 0.959 & 1.000 & 0.998 & 1.000 & 1.000 \\
\hline M11 & 0.452 & 0.309 & 0.813 & 0.442 & 0.714 & 0.927 & 0.703 & 0.404 \\
\hline M12 & 0.635 & 0.460 & 0.867 & 0.689 & 0.735 & 0.868 & 0.782 & 0.597 \\
\hline M13 & 0.687 & 0.240 & 0.813 & 0.742 & 0.608 & 0.790 & 0.751 & 0.885 \\
\hline M14 & 0.600 & 0.721 & 0.867 & 0.647 & 0.755 & 0.891 & 0.759 & 0.438 \\
\hline
\end{tabular}

Secondly, we calculate each node's performance value in eight indicators; the results are as shown in Table 3, and the normalized results are as shown in Table 4.

Thirdly, using (22), we can obtain the weighted normalized decision matrix as follows:

V

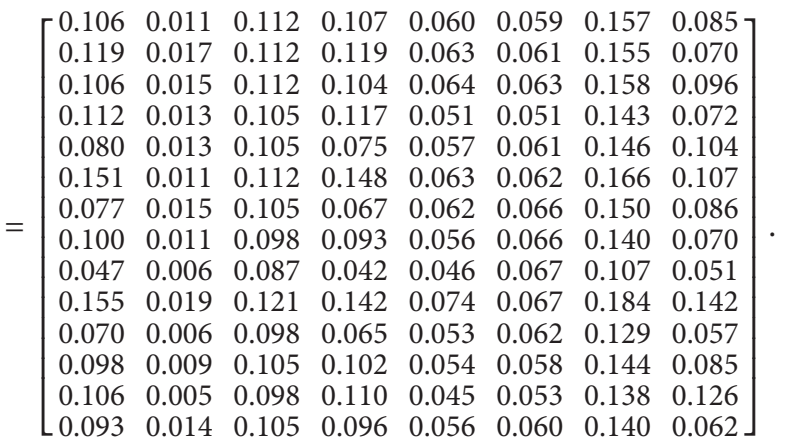

The positive-ideal node $A^{+}$is

$\left\{\begin{array}{llllllll}0.155 & 0.019 & 0.121 & 0.148 & 0.074 & 0.067 & 0.184 & 0.142\end{array}\right.$.

The negative-ideal node $A^{-}$is

$\left\{\begin{array}{llllllll}0.070 & 0.005 & 0.087 & 0.042 & 0.045 & 0.053 & 0.107 & 0.051\end{array}\right.$.

Finally, use (24) to calculate the distances from the positive-ideal node $A^{+}$and the negative-ideal node $A^{-}$. Take the member 1 as the example:

$$
d_{1}^{+}=0.092 ; \quad d_{1}^{-}=0.111
$$

Use (25) to calculate the closeness coefficient of the member 1:

$$
\begin{aligned}
& Z_{1}^{+}=\frac{0.092}{0.092+0.111}=0.453 \\
& Z_{1}^{-}=\frac{0.111}{0.092+0.111}=0.547
\end{aligned}
$$


TABLE 5: Closeness coefficients to aspired level among different nodes.

\begin{tabular}{lccccc}
\hline & $d_{i}^{+}$ & $d_{i}^{-}$ & $Z_{i}^{+}$ & $Z_{i}^{-}$ & 0.547 \\
M1 & 0.092 & 0.111 & 0.453 & 0.570 & 0.575 \\
M2 & 0.092 & 0.122 & 0.430 & 0.519 & 5 \\
M3 & 0.085 & 0.115 & 0.425 & 0.412 & 7 \\
M4 & 0.110 & 0.481 & 0.801 & 10 \\
M5 & 0.102 & 0.084 & 0.588 & 0.359 & 2 \\
M6 & 0.120 & 0.074 & 0.199 & 0.417 & 0.066 \\
M7 & 0.043 & 0.085 & 0.641 & 0.970 & 12 \\
M8 & 0.132 & 0.014 & 0.583 & 0.217 & 1 \\
M1 & 0.119 & 0.196 & 0.934 & 0.478 & 0.030 \\
M11 & 0.198 & 0.044 & 0.783 & 0.578 \\
M12 & 0.006 & 0.122 & 0.522 & 0.396 \\
\hline
\end{tabular}

Similarly, we can obtain the other thirteen nodes' closeness coefficients as shown in Table 5.

From Table 5, we can see the importance degree and gap degree of each node. The importance degrees of 14 team members, respectively, are $0.547,0.570,0.575,0.519,0.412$, $0.801,0.359,0.417,0.066,0.970,0.217,0.478,0.578$, and 0.396 . In the fourteen nodes, the importance degree of node 10 is the biggest, and node 6 is also one of the most important two nodes in this team; that is, the distances of their importance degree to the positive-ideal node are 0.003 and 0.199 , respectively. We can think that they can substantially affect the team activities and other members behaviors. By further investigation, we found that the member of node 10 is the leader of this product R\&D team; he has the greatest power conferred by his position in the company and needs to communicate and exchange with every member in the team. Serving as "bridges" in most structural holes position, he controls most organizational information and knowledge resources. The leader is indeed the most important node in complex network of product R\&D team.

Furthermore, we found the member of node 6 is the opinion leader in this product $\mathrm{R} \& \mathrm{D}$ team. The years he severed in this company are the longest, and he has a wealth of experience in product development and creative activities. In the creative solution formation process of the front-end innovation, when the other members generate the new ideas, they are accustomed to seek his advice, and when they meet some difficult things in work, they like to seek help from him. So the member of node 6 is the second most important node in this team, which is consistent with our analysis results.

The members of nodes $1,2,3,4$, and 13 have the relative strong importance degree in the complex networks of this product $\mathrm{R} \& \mathrm{D}$ team; they are the key members engaged in generating and developing creative ideas or creative solutions, which is the core work in the whole product innovation process. The members of nodes $5,7,8,11,12$, and 13 are less important than the members previously mentioned, and the member of node 9 has the least importance degree in the team, who rarely participates in team work. Hence, in the actual management, the company leader and management can focus more on the members of nodes $1,2,3,4,6,10$, and 13 , who are directly related to the team results.

\section{Conclusions}

Assessing the node importance of complex networks in product $\mathrm{R} \& \mathrm{D}$ team and seeking the member who is the most important in the team is particularly important to the companies engaged in creativity and innovation. Pervious studies have developed and established some methods in order to solve these issues, and they propose many assessment indicators. However, when we are assessing the node importance of complex network, we cannot give out the results depending on several separate variable; we need to comprehensively evaluate the node importance simultaneously taking into account a number of indicators. In this paper, we choose eight indicators from two aspects: centralization and structural holes of complex networks, such as degree centrality, betweenness centrality, closeness centrality, eigenvector centrality, effective size, efficiency, constraint, and hierarchy. We combine the fuzzy AHP and TOPSIS and establish the multiple attribute decision making model to help us seek the important nodes of complex networks. Then we took a product $\mathrm{R} \& \mathrm{D}$ team of a game software company as a research example; we used the model we established to seek the important members in this team and successfully find out the important members who are consistent with the fact. We confirmed the effectiveness, operability, and efficiency of the method used to seek the important nodes of complex networks.

\section{Conflict of Interests}

The authors do not have any conflict of interests with the content of the paper.

\section{Acknowledgment}

This research is supported by the National Natural Science Foundation of China (Grant no. 71273076), Humanities and 
Social Sciences Youth Foundation of Chinese Ministry of Education (Grant no. 3YJC630166), and the Natural Science Foundation of Heilongjiang Province of China (Grant no. G201006).

\section{References}

[1] J. Goldenberg, D. R. Lehmann, and D. Mazursky, “The idea itself and the circumstances of its emergence as predictors of new product success," Management Science, vol. 47, no. 1, pp. 69-84, 2002.

[2] M. J. Garfield, N. J. Taylor, A. R. Dennis, and J. W. Satzinger, "Research report: modifying paradigms-individual differences, creativity techniques, and exposure to ideas in group idea generation," Information Systems Research, vol. 12, no. 3, pp. 322-333, 2001.

[3] H. Gao, J. Lam, and G. Chen, "New criteria for synchronization stability of general complex dynamical networks with coupling delays," Physics Letters A, vol. 360, no. 2, pp. 263-273, 2006.

[4] Z. Wang, Y. Wang, and Y. Liu, "Global synchronization for discrete-time stochastic complex networks with randomly occurred nonlinearities and mixed time delays," IEEE Transactions on Neural Networks, vol. 21, no. 1, pp. 11-25, 2010.

[5] Y. C. Lai, A. E. Motter, and T. Nishikawa, Attacks and Cascades in Complex Networks, Springer, Berlin, Germany, 2004.

[6] H. Chen, W. Chung, J. J. Xu, G. Wang, Y. Qin, and M. Chau, "Crime data mining: a general framework and some examples," Computer, vol. 37, no. 4, pp. 50-56, 2004.

[7] J. Golbeck and J. A. Hendler, "Reputation network analysis for Email filtering," in Proceedings of the 1st Conference on Email and Anti-Spam, Mountain View, Calif, USA, 2004.

[8] J. Shetty and J. Adibi, "Discovering important nodes through graph entropy the case of enron email database," in Proceedings of the 3rd International Workshop on Link Discovery, pp. 74-81, ACM, 2005.

[9] T. Opsahl, F. Agneessens, and J. Skvoretz, "Node centrality in weighted networks: generalizing degree and shortest paths," Social Networks, vol. 32, no. 3, pp. 245-251, 2010.

[10] D. J. Watts, P. S. Dodds, and M. E. J. Newman, "Identity and search in social networks," Science, vol. 296, no. 5571, pp. 13021305, 2002.

[11] R. Guimera, S. Mossa, A. Turtschi, and L. A. N. Amaral, "The worldwide air transportation network: anomalous centrality, community structure, and cities' global roles," Proceedings of the National Academy of Sciences of the United States of America, vol. 102, no. 22, pp. 7794-7799, 2005.

[12] A. Broder, R. Kumar, F. Maghoul et al., "Graph structure in the Web," Computer Networks, vol. 33, no. 1, pp. 309-320, 2000.

[13] D. H. Zanette, "Dynamics of rumor propagation on small-world networks," Physical Review E, vol. 65, no. 4, Article ID 041908, 2002.

[14] Y. Tang, H. Gao, and W. Zou, "Identifying controlling nodes in neuronal networks in different scales," PloS ONE, vol. 7, Article ID e41375, 2012.

[15] Y. Tang, Z. Wang, H. Gao, S. Swift, and J. Kurths, "A constrained evolutionary computation method for detecting controlling regions of cortical networks," IEEE/ACM Transactions on Computational Biology and Bioinformatics, vol. 9, pp. 1569-1581, 2012.
[16] B. Shen, Z. Wang, and X. Liu, "Sampled-data synchronization control of complex dynamical networks with stochastic sampling," IEEE Transactions on Automatic Control, vol. 57, pp. 2644-2650, 2012.

[17] J. Hu, Z. Wang, H. Gao, and L. K. Stergioulas, "Extended Kalman filtering with stochastic nonlinearities and multiple missing measurements," Automatica, vol. 48, pp. 2007-2015, 2012.

[18] D. Ding, Z. Wang, H. Dong, and H. Shu, "Distributed H-infinity state estimation with stochastic parameters and nonlinearities through sensor networks: the finite-horizon case," Automatica, vol. 48, pp. 1575-1585, 2012.

[19] D. Ding, Z. Wang, B. Shen, and H. Shu, "H-infinity state estimation for discrete-time complex networks with randomly occurring sensor saturations and randomly varying sensor delays," IEEE Transactions on Neural Networks and Learning Systems, vol. 23, pp. 725-736, 2012.

[20] B. Shen, Z. Wang, and X. Liu, "Bounded $H_{\infty}$ synchronization and state estimation for discrete time-varying stochastic complex networks over a finite horizon," IEEE Transactions on Neural Networks, vol. 22, no. 1, pp. 145-157, 2011.

[21] D. B. West, Introduction to Graph Theory, Prentice Hall, Englewood Cliffs, NJ, USA, 2001.

[22] T. Washio and H. Motoda, "State of the art of graph-based data mining," ACM SIGKDD Explorations Newsletter, vol. 5, pp. 5968, 2003.

[23] D. Knoke and R. S. Burt, "Prominence," in Applied Network Analysis, pp. 195-222, Sage, 1983.

[24] H. Nan, L. Deyi, and G. Wenyan, "Mining vital nodes in complex networks," Computer Science, vol. 34, pp. 1-5, 2007.

[25] T. H. Grubesic and A. T. Murray, "Vital nodes, interconnected infrastructures, and the geographies of network survivability," Annals of the Association of American Geographers, vol. 96, no. 1, pp. 64-83, 2006.

[26] S. Brin and L. Page, "The anatomy of a large-scale hypertextual Web search engine 1," Computer Networks, vol. 30, no. 1-7, pp. 107-117, 1998.

[27] J. M. Kleinberg, "Authoritative sources in a hyperlinked environment," Journal of the ACM, vol. 46, no. 5, pp. 604-632, 1999.

[28] Y. Hui, L. Zun, and L. Yong-Jun, "Key nodes in complex networks identified by multi-attribute decision-making method," Acta Physica Sincia, vol. 62, Article ID 020204, 2013.

[29] R. S. Burt, Structural Holes: The Social Structure of Competition, Harvard University Press, 2009.

[30] L. A. Zadeh, "Fuzzy sets," Information and Control, vol. 8, no. 3, pp. 338-353, 1965.

[31] G. Büyüközkan, O. Feyzioğlu, and E. Nebol, "Selection of the strategic alliance partner in logistics value chain," International Journal of Production Economics, vol. 113, pp. 148-158, 2008.

[32] T.-Y. Hsieh, S.-T. Lu, and G.-H. Tzeng, "Fuzzy MCDM approach for planning and design tenders selection in public office buildings," International Journal of Project Management, vol. 22, no. 7, pp. 573-584, 2004.

[33] W. Zhang and Q. Zhang, "Multi-stage evaluation and selection in the formation process of complex creative solution," Quality \& Quantity, 2013.

[34] J. J. Buckley, "Fuzzy hierarchical analysis," Fuzzy Sets and Systems, vol. 17, no. 3, pp. 233-247, 1985.

[35] C.-T. Chen, C.-T. Lin, and S.-F. Huang, "A fuzzy approach for supplier evaluation and selection in supply chain management," International Journal of Production Economics, vol. 102, no. 2, pp. 289-301, 2006. 


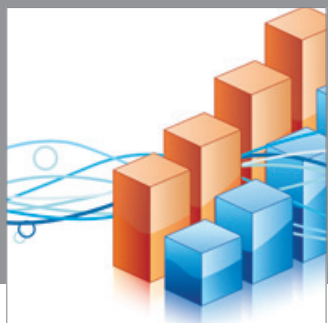

Advances in

Operations Research

mansans

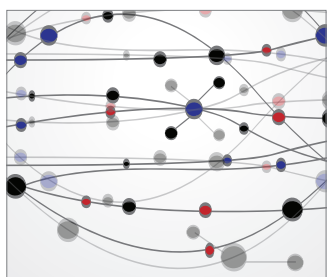

The Scientific World Journal
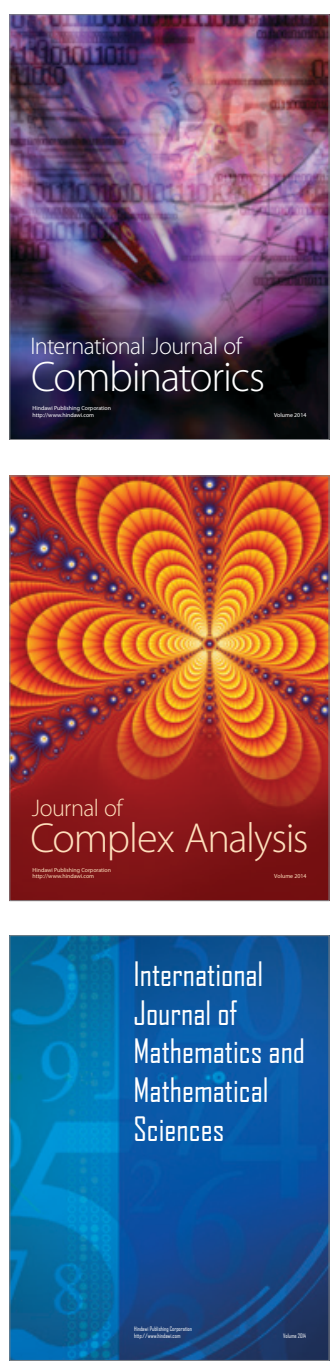
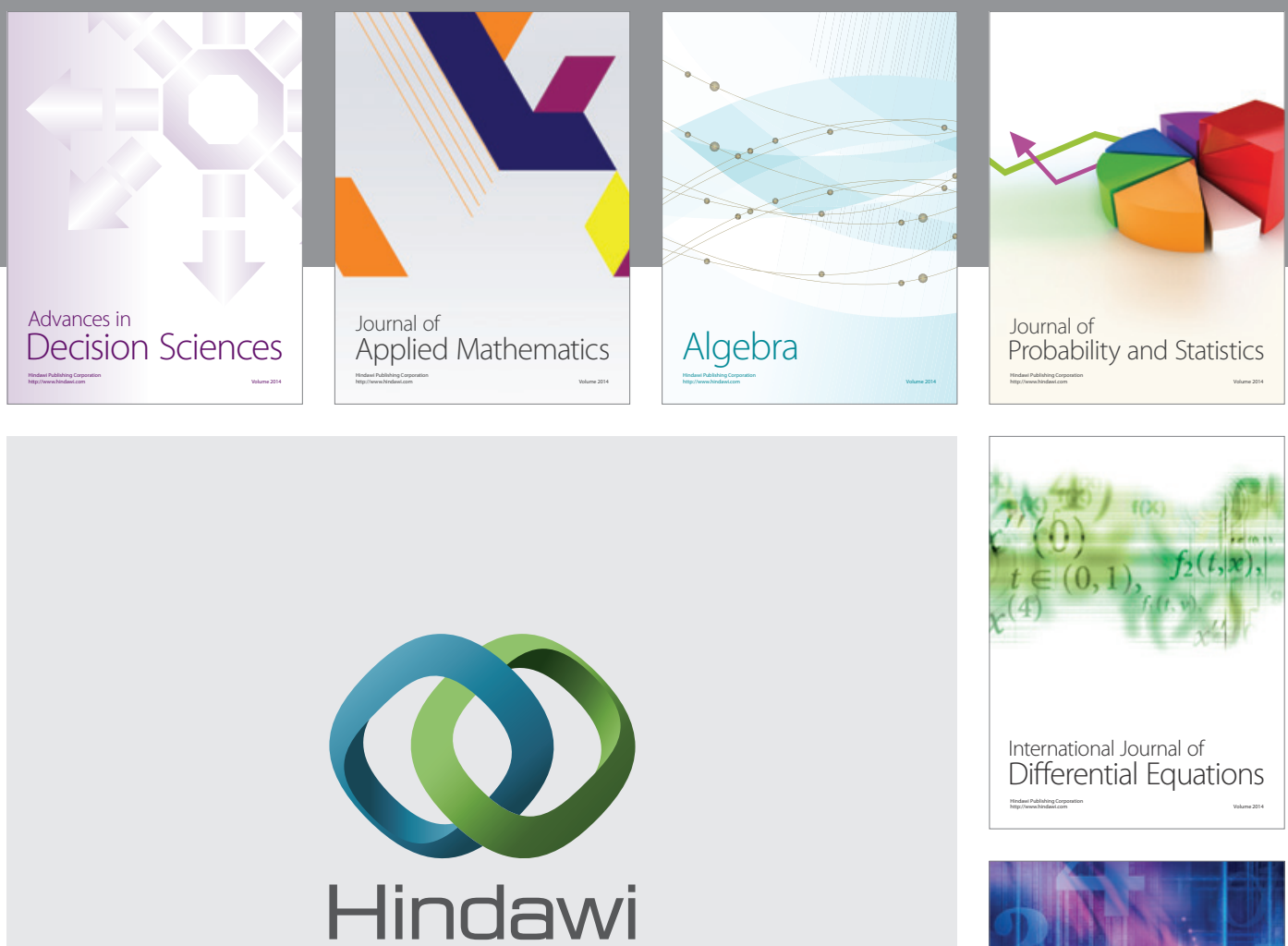

Submit your manuscripts at http://www.hindawi.com
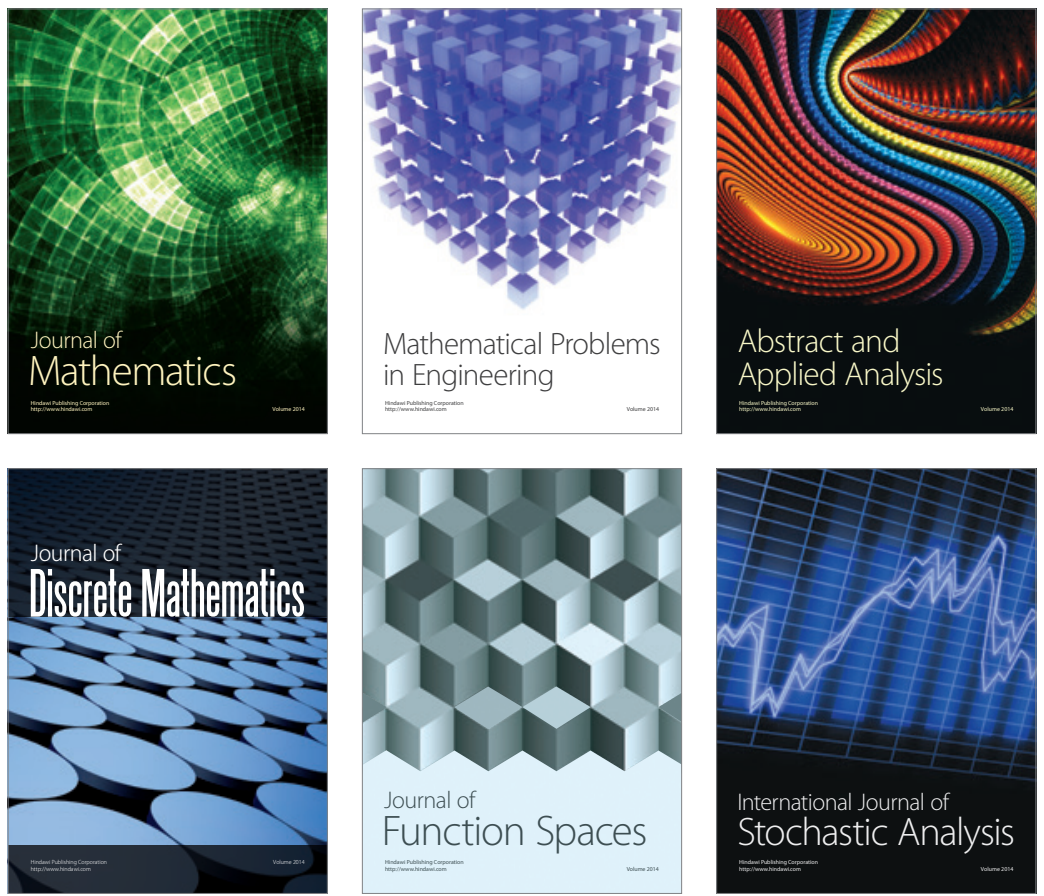

Journal of

Function Spaces

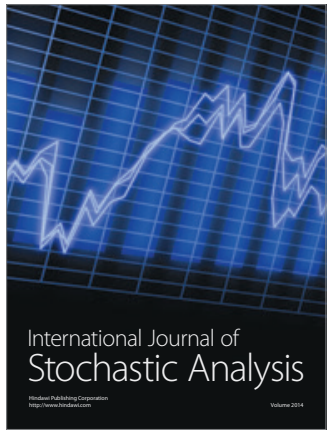

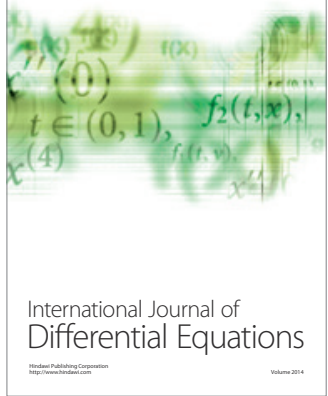
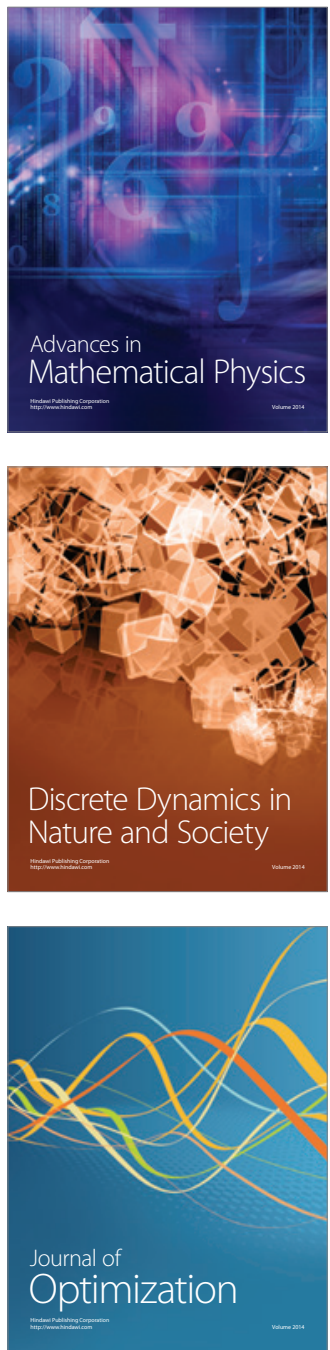